\title{
Solvent free interactions in contact pairs of molecules of biological interest: Laser spectroscopic and electrospray mass spectrometric studies
}

\author{
D. Scuderi, ${ }^{1,2}$ A. Paladini, ${ }^{1}$ M. Satta, ${ }^{4}$ D. Catone,,${ }^{1}$ F. Rondino, ${ }^{1}$ A. Filippi, ${ }^{2}$ \\ S. Piccirillo, ${ }^{3}$ M. Speranza, ${ }^{2}$ and A. Giardini Guidoni ${ }^{1,4, \dagger}$ \\ ${ }^{1}$ Dipartimento di Chimica, Università di Roma "La Sapienza", pl. A. Moro 5, I-00185 Roma, Italy \\ ${ }^{2}$ Facoltà di Farmacia, Dipartimento di Studi di Chimica e Tecnologia delle Sostanze Biologicamente Attive, \\ Università di Roma "La Sapienza", pl. A. Moro 5, I-00185 Roma, Italy \\ ${ }^{3}$ Università di Roma "Tor Vergata” Dipartimento di Scienze e Tecnologie Chimiche, \\ Via della Ricerca Scientifica, I-00133 Rome, Italy \\ ${ }^{4}$ CNR-IMIP (sezione Istituto Materiali Speciali), I-85050 Tito Scalo (Pz), Italy
}

\begin{abstract}
A laser spectroscopic and mass spectrometric study of ionic and molecular clusters of biological interest is reported. The molecules of interest and their aggregates were generated in a supersonic beam and analyzed by mass resolved resonant two photon absorption and ionization (R2PI) and by collision induced mass spectrometry (CID-MS). The absence of the solvent allows to study these systems in the isolated state free of undesired solvent effects which may level off the differences in their properties. The gas phase results have been compared to theoretical estimates of the structure and stability of the systems under investigation.
\end{abstract}

\section{INTRODUCTION}

In recent years, molecular-scale studies on isolated clusters of biological relevance were allowed by the development of gas phase methodologies such as laser spectroscopy and mass spectrometry, which provided many important pieces of information about the cluster structure, ionization potential, intermolecular forces, and stability [1]. Only recently, these methodologies have been applied to the study of chiral systems [2, 3]. In particular, Electrospray Ionization Mass Spectrometry (ESI-MS) [4-6] and Resonance Enhanced MultiPhoton Ionization [3, 7] coupled with time-of-flight mass spectrometry (REMPI-TOF), proved most useful for this purpose. Both techniques rely on the measure of the different stability of diastereomeric complexes arising from the combination of a chiral molecule with a chiral selector. Recent R2PI studies, performed in our group, provided some insight into the spectroscopic shifts and the binding energies of aromatic chromophores (C) with solvent molecules, including chirals, which can be attributed to the interplay between the various forces acting between them $[1,7,8]$. Advanced theoretical computations revealed very helpful in recognizing the role played by the electrostatic and hydrogen bonding interactions in these systems.

This paper is aimed at reporting results of a comprehensive study on the enantiodiscrimination of chiral molecules in the gas phase through the application of REMPI-TOF methodologies and the ESI-MS ${ }^{n}$ Collision

\footnotetext{
†E-mail: anna.giardini@uniroma1.it
}

Induced Dissociation (CID). The mass resolved R2PI spectra of diastereomeric clusters of (R)-(+)-1-phenyl1-propanol $\left(\mathbf{P}_{\mathbf{R}}\right)$ and $(\mathrm{R})-(-)$-1-indanol $\left(\mathbf{I}_{\mathbf{R}}\right)$ with (R)-(-)and $(\mathrm{S})-(+)-2$-hexanol $\left(\mathbf{X}_{\mathbf{R}}\right.$ and $\left.\mathbf{X}_{\mathbf{S}}\right)$ are discussed. The ESI-MS ${ }^{n}$-CID measurements concern trimeric clusters ions $\left[\mathbf{M} \cdot(\boldsymbol{r} \boldsymbol{e})_{2} \cdot \mathbf{S}\right]^{+}$between first-group metal ions $\left(\mathbf{M}^{+}\right)$and chiral $\alpha$-aminophosphonic acids (ref and $\mathbf{S}$ ). The $\alpha$-aminophosphonic acids investigated are $(R)-(-)$ and $(S)-(+)-\left(1\right.$-aminoethyl) phosphonic acid (ame $\mathbf{R}_{\mathbf{R}}$ and ame $\left._{S}\right),(1 R)-(+)-$ and (1S)-(-)-(1-amino-2-methyl propyl) phosphonic acid $\left(\operatorname{amp}_{\mathrm{R}}\right.$ and $\left.\mathbf{a m p}_{\mathrm{S}}\right)$.

\section{EXPERIMENTAL METHODOLOGIES}

2.1. Resonant Multi-Photon Ionisation Spectroscopy (REMPI-TOF). The experimental set-up, which combines a supersonic molecular beam, two Nd-YAG pumped dye lasers and a time of flight (TOF) mass spectrometer, has been elsewhere described [1, 9]. Supersonic beam production of the adducts is obtained by adiabatic expansion of a carrier gas (Ar) seeded with a selected chromophore (C) and solvent molecule (solv) through a heatable pulsed nozzle of $400 \mu \mathrm{m}$ i.d. The concentration is maintained enough low to minimize the production of heavier clusters. The skimmed supersonic jet ( $1 \mathrm{~mm}$ skimmer diameter) enters into a second chamber equipped with a TOF mass spectrometer. Molecules and clusters in the beam are excited and ionized by one or two tunable dye lasers and the ionized species are detected by a channeltron. The mass selected photoionization signals are recorded and averaged by a digital oscilloscope and stored on a PC. 


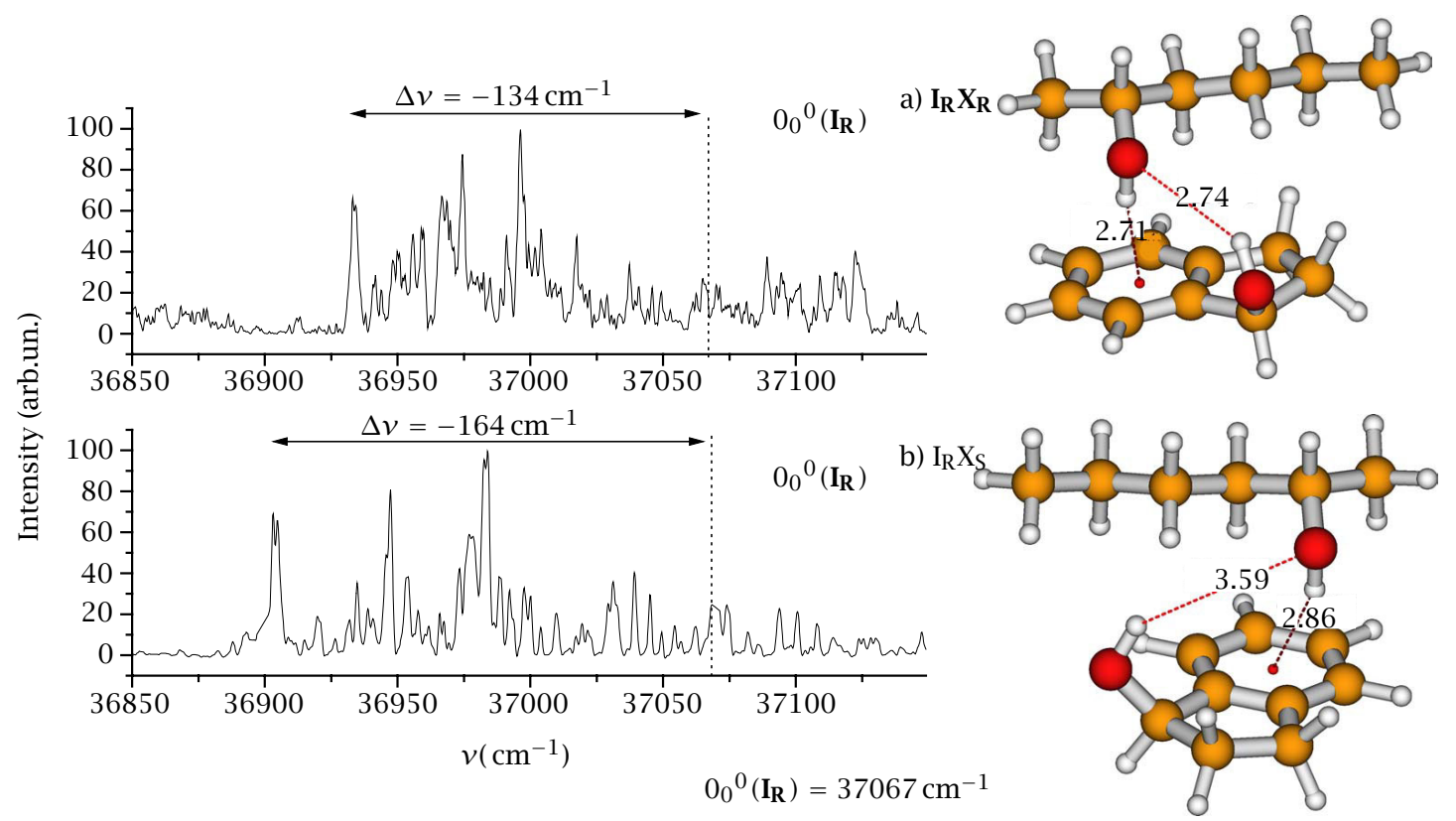

Figure 1. 1cR2PI excitation spectra of diastereomeric complexes of $\mathbf{I}_{\mathbf{R}}$ with a) $\mathbf{X}_{\mathbf{R}}$ and $\left.b\right) \mathbf{X}_{\mathbf{S}}$ taken at $m / z=236$ and at total stagnation pressure of $2 \times 10^{5} \mathrm{~Pa}$. Their structures calculated using the Molecular Mechanical approach following a basin hopping search model based on the MM3 force field are reported as well.

One color R2PI experiments (1cR2PI) involve electronic excitation of the species of interest by absorption of one photon $h v_{1}$ and ionization by absorbing a second photon $h v_{1}$. Mass discrimination of the ionized complex may be complicated by fragmentation processes, due to the excess energy gained during ionization. The ionization and fragmentation thresholds have been obtained by the photoionization efficiency curves through a two color R2PI (2cR2PI) sequence: i- the first exciting laser $\left(h v_{1}\right)$ is tuned on the $\mathrm{S}_{1} \leftarrow \mathrm{S}_{0}$ transition of the species of interest; ii- the laser intensity is lowered to about $1 \%$ of the initial fluence to minimize the $h v_{1}$ absorption; iii- a second laser $\left(h v_{2}\right)$ is scanned through the cluster ionization and fragmentation threshold regions. The photoionization spectra were corrected for the effect of the electric field strength $(200 \mathrm{~V} / \mathrm{cm})$ produced by the extraction plates of the TOF spectrometer. The binding energy $\mathrm{D}_{0}$ of the $\mathbf{C} \cdot$ solv adduct is computed from the difference between its dissociative ionization threshold $A E(\mathbf{C})^{+}=h v_{1}(\mathbf{C} \cdot \text { solv })^{*}+h v_{2}\left(\mathbf{C}^{+}+\right.$solv $)$ and the ionization threshold of the bare $\mathbf{C}$, i.e. $I P(\mathbf{C})=$ $h v_{1}(\mathbf{C})^{*}+h v_{2}(\mathbf{C})^{+}$. The dissociation energy $\mathrm{D}^{+}{ }_{0}$ of the ionic cluster $(\mathbf{C} \cdot \boldsymbol{s o l v})^{+}$is calculated from the difference between its dissociative ionization threshold, $A E(\mathbf{C})^{+}=$ $h v_{1}(\mathbf{C} \cdot \text { solv })^{*}+h v_{2}\left(\mathbf{C}^{+}+\right.$solv $)$, and its ionization threshold $I P(C \cdot$ solv $)=h v_{1}(\mathbf{C} \cdot \text { solv })^{*}+h v_{2}(C \cdot \text { solv })^{+}$.

\subsection{Electrospray Ionisation Mass Spectrometry}

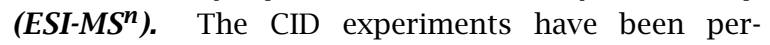
formed by using both a commercial API 100/300 triplequadrupole $(\mathrm{QqQ})$ mass spectrometer from Perkin Elmer Sciex, equipped with an ESI source and a syringe pump [10]. Operating conditions for the ESI source have been as follows: spray voltage, $3.8 \mathrm{KV}$; capillary temperature, $298 \mathrm{~K}$; sheath gas $\left(\mathrm{N}_{2}\right)$ flow rate, 30 units (roughly $0.75 \mathrm{~L} / \mathrm{min}$ ). The selected gas phase complexes have been generated by electrospraying at a flow rate of $10 \mu \mathrm{l} / \mathrm{min}$ 50/50 water/methanol solutions containing equimolar amounts (10 $\mu \mathrm{M}$ each) of the optically pure solvent molecule and a chiral reference compound. The CID experiments on the so-formed diastereomeric complexes have been conducted in the positive ion mode. In the full scan QqQ-MS ${ }^{2}$ mode, the diastereomeric cluster ions have been isolated in the first mass analysing quadrupole $\mathrm{Q}$, excited in the second "rf-only" quadrupole q by collision with $\mathrm{N}_{2}$ (pressure ca. 10 mbar; energy range $6-12 \mathrm{eV}$ in the laboratory frame), and eventually analyzed in the third quadrupole $\mathrm{Q}$ of the instrument.

\section{RESULTS AND DISCUSSIONS}

\subsection{R2PI enantiodifferentiation of chiral mole-}

cules. Enantiodifferentiation of neutral chiral molecules in the gas phase can be obtained by mass resolved R2PI spectroscopy of their adducts with chiral selectors. Diastereomeric pairs are characterized by the non equivalence of their interaction energy in both the ground and excited state and therefore they exhibit different spectroscopic properties [3, 7]. Figure 1 reports the $1 \mathrm{cR} 2 \mathrm{PI}$ excitation spectra of the diastereomeric complexes of $\mathbf{I}_{\mathbf{R}}$ with $\mathbf{X}_{\mathbf{R}}$ and $\mathbf{X}_{\mathbf{S}}$ together with structures predicted using Molecular Mechanical approach following a basin hopping search 


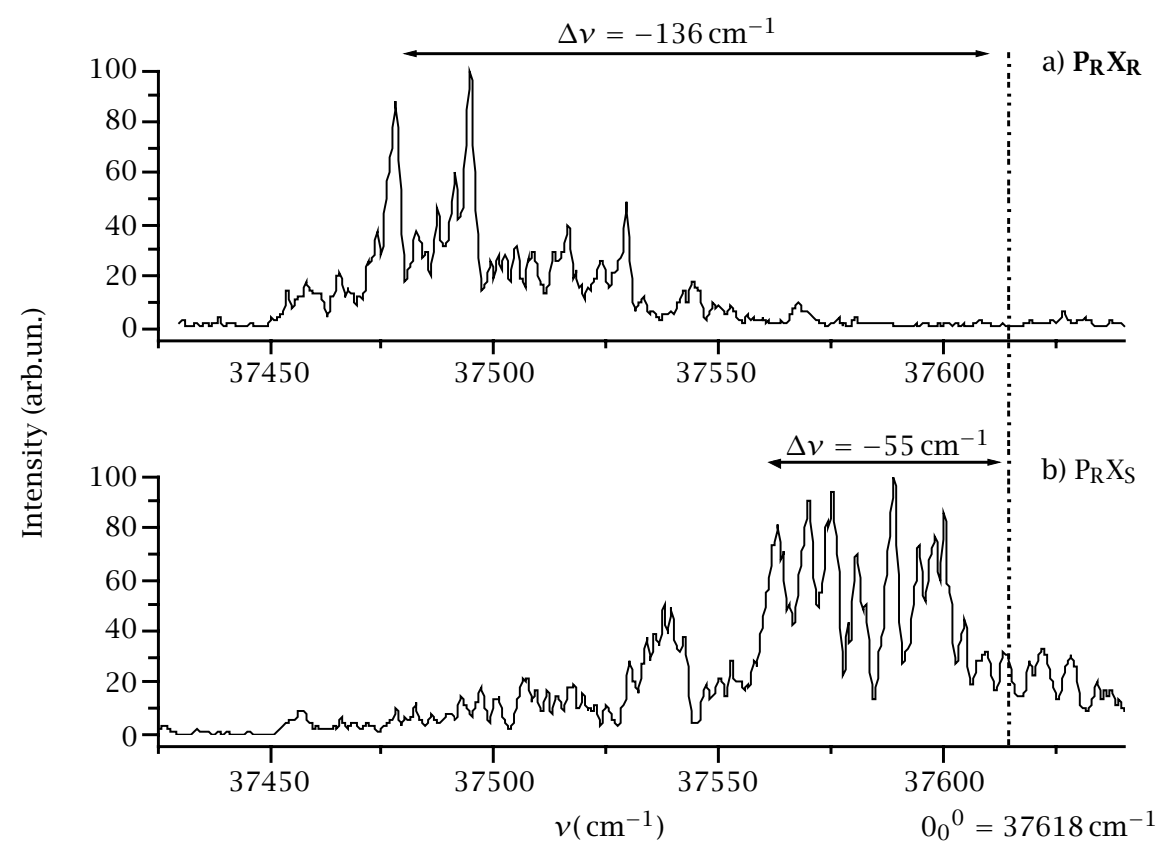

Figure 2. 1cR2PI excitation spectra of diastereomeric complexes of $\mathbf{P}_{\mathbf{R}}$ with a) $\mathbf{X}_{\mathbf{R}}$ and $\left.b\right) \mathbf{X}_{\mathbf{S}}$ taken at $m / z=238$ and at total stagnation pressure of $2 \times 10^{5} \mathrm{~Pa}$.

model based on the MM3 force field [11]. The evident difference in the spectral features provides a further example of enantiodifferentiation of chiral $\mathbf{X}_{\mathbf{R}}$ and $\mathbf{X}_{\mathbf{S}}$ alcohols by this methodology. The spectra are complicated by vibronic transitions associated with the many conformers of the adducts. As already observed for other chiral clusters, both $\left[\mathbf{I}_{\mathbf{R}} \cdot \mathbf{X}_{\mathbf{R}}\right]$ and $\left[\mathbf{I}_{\mathbf{R}} \cdot \mathbf{X}_{\mathbf{S}}\right]$ complexes exhibit spectral signatures characterized by a significant red shift $\Delta v$ of the $0_{0}{ }^{0} \mathrm{~S}_{1} \leftarrow \mathrm{S}_{0}$ transition relative to that of the bare chromophore $\mathbf{I}_{\mathbf{R}}$ [12]. The spectral shifts reflect the combined effect of the electrostatic and dispersive interactions between C and solv on the HOMO and LUMO energies of the chromophore.

The homochiral $\left[\mathbf{I}_{\mathbf{R}} \cdot \mathbf{X}_{\mathbf{R}}\right]$ cluster displays a red shift $\left(\Delta \nu_{\text {homo }}\right)$ less negative than that of the heterochiral analogue $\left[\mathbf{I}_{\mathbf{R}} \cdot \mathbf{X}_{\mathbf{S}}\right]\left(\Delta \nu_{\text {hetero }}\right)$. The $\Delta(\Delta v)=\Delta v_{\text {homo }}-$ $\Delta v_{\text {hetero }}=+30 \mathrm{~cm}^{-1}$ difference is consistent with the hypothesis of a $S_{1} \leftarrow S_{0}$ energy gap of the heterochiral complex smaller than that of the corresponding homochiral adduct.

Figure 2 reports the $1 \mathrm{cR} 2 \mathrm{PI}$ excitation spectra of the diastereomeric complexes of $\mathbf{P}_{\mathbf{R}}$ with $\mathbf{X}_{\mathbf{R}}$ and $\mathbf{X s}$. The homochiral $\left[\mathbf{P}_{\mathbf{R}} \cdot \mathbf{X}_{\mathbf{R}}\right]$ cluster displays a red shift $\left(\Delta \nu_{\text {homo }}\right)$ more negative than that of the heterochiral analogue $\left[\mathbf{P}_{\mathbf{R}} \cdot \mathbf{X}_{\mathbf{S}}\right]\left(\Delta v_{\text {hetero }}\right)$. The $\Delta(\Delta v)=\Delta v_{\text {homo }}-\Delta v_{\text {hetero }}=$ $-81 \mathrm{~cm}^{-1}$ difference is consistent with the hypothesis of a $S_{1} \leftarrow S_{0}$ energy gap of the homochiral complex smaller than that of the corresponding heterochiral adduct. This trend is opposite to that of $\left[\mathbf{I}_{\mathbf{R}} \cdot \mathbf{X}_{\mathbf{R}}\right]$ and $\left[\mathbf{I}_{\mathbf{R}} \cdot \mathbf{X}_{\mathbf{S}}\right]$ pairs and parallels that found for the clusters of
$\mathbf{P}_{\mathbf{R}}$ with (R)-(-) and (S)-(+) 2-pentanol $\left(\mathbf{T}_{\mathbf{R}}\right.$ and $\left.\mathbf{T}_{\mathbf{S}}\right)$. The opposite sign of the $\Delta(\Delta v)$ values supports the view that the HOMO-LUMO gap in $\left[\mathbf{P}_{\mathbf{R}} \cdot \boldsymbol{s o l v}\right]$ complexes with secondary alcohols is considerably affected by steric factors.

In the 1cR2PI experiments, an excess vibrational energy is absorbed by the ionic adduct which undergoes extensive fragmentation. The spectral patterns of the fragment ions are found to be equal to that of the parent ion. Ionization of the diastereomeric $\left[\mathbf{I}_{\mathbf{R}} \cdot \mathbf{X}_{\mathbf{R}}\right]$ and $\left[\mathbf{I}_{\mathbf{R}} \cdot \mathbf{X}_{\mathbf{S}}\right]$ complexes gives rise to the same fragmentation pattern mainly characterized by the loss of solv, although fragmentation is comparatively less extensive for the homochiral adduct. A fragmentation characterized also by loss of ethyl group from the chromophore $\mathbf{C}$, is found for $\left[\mathbf{P}_{\mathbf{R}} \cdot \mathbf{X}_{\mathbf{R}}\right]$ and $\left[\mathbf{P}_{\mathbf{R}} \cdot \mathbf{X}_{\mathbf{S}}\right]$. These data parallel those found for other diasteromeric complexes with $\mathbf{C}=\mathbf{T}_{\mathbf{R}}, \mathbf{E}_{\mathbf{R}}$ or $\mathbf{P}_{\mathbf{R}}$, whose measured binding energies are generally larger in the homochiral complexes. The $\Delta(\Delta v)$ values and the highly reproducible differences in the R2PI fragmentation pattern of diastereomeric clusters represent useful tools for the enantiodiscrimination of chiral molecules in the gas phase and provide some insights into the balance between attractive and steric repulsive forces operating in these systems.

\subsection{ESI-MS ${ }^{2}-C I D$ enantiodifferentiation of some phosphorous-containing $\alpha$-amino acid mimics.} This section deals with the application of the ESI-MS ${ }^{2}$ CID technique to the enantiodiscrimination of several chiral $\alpha$-aminophosphonic acids. $\alpha$-Aminophosphonic 
acids are the analogues of natural $\alpha$-aminocarboxylic acids present in biological systems. The biological activity of these chiral compounds is governed by the stability of their inclusion complexes into the chiral enzyme cavity, specifically with its metal ion centers. For this reason, we deemed it important to investigate the affinity of $\alpha$-aminophosphonic acids towards firstgroup metals in gaseous phase and how this can be affected by the ligand configuration. The results are discussed in the light of structure calculation performed by using an empirical force field.

According to the foundations of Cooks' kinetic method [4], gas phase discrimination of chiral solvent $\mathrm{S}_{\mathrm{R}}$ and $\mathrm{S}_{\mathrm{S}}$ is obtained by measuring of the relative stability of their diastereomeric complexes with a chiral reference selector ref. CID of the diastereomeric cluster ions $\left[\mathbf{M} \cdot(\boldsymbol{r e f})_{2} \cdot \mathbf{S}_{\mathbf{R}}\right]^{+}$and $\left[\mathbf{M} \cdot(\boldsymbol{r e f})_{2} \cdot \mathbf{S}_{\mathbf{S}}\right]^{+}$, where $\mathbf{M}^{+}=\mathrm{H}^{+}, \mathrm{Li}^{+}, \mathrm{Na}^{+}$and $\mathrm{K}^{+}$, may produce different fragmentation patterns reflecting the relevant $\left[\mathbf{M} \cdot \boldsymbol{r e f} \cdot \mathbf{S}_{\mathbf{R}}\right]^{+}$ and $\left[\mathbf{M} \cdot \boldsymbol{r e f} \cdot \mathrm{S}_{\mathbf{S}}\right]^{+}$vs. $\left[\mathbf{M} \cdot(\boldsymbol{r e f})_{2}\right]^{+}$stability. It is convenient to define the cluster ions as "homo" when $\mathbf{S}$ and ref have the same configuration, and "hetero" in the opposite case. Measurement of the ratio of the "homo" vs. "hetero" ion abundance ratios provides the chiral selectivity $\mathbf{R}_{\text {chiral }}$, i.e.:

$$
\mathbf{R}_{\text {chiral }}=\frac{\mathbf{R}_{\text {HOMO }}}{\mathbf{R}_{\text {HETERO }}}=\frac{\left[\mathbf{M} \cdot \operatorname{ref} \cdot \mathbf{S}_{\mathbf{S}}\right]^{+} /\left[\mathbf{M} \cdot(\text { ref })_{2}\right]^{+}}{\left[\mathbf{M} \cdot \operatorname{ref} \cdot \mathbf{S}_{\mathbf{R}}\right]^{+} /\left[\mathbf{M} \cdot(\mathbf{r e f})_{2}\right]^{+}}
$$

if the $\mathbf{S}$ enantiomer of $\boldsymbol{r} \boldsymbol{e} \boldsymbol{f}$ is employed.

Typical CID fragmentation spectra of diastereomeric clusters containing one of the $\mathbf{a m p}_{\mathrm{R}}$ and $\mathbf{a m p}_{\mathrm{S}}$ enantiomers, as solvent, ames as ref, and a sodium ion $\left(\left[\mathrm{Na} \cdot\left(\mathbf{a m e}_{\mathbf{S}}\right)_{2} \cdot\left(\mathbf{a m p}_{\mathbf{R}}\right)\right]^{+}\right.$and $\left.\left[\mathrm{Na} \cdot\left(\mathbf{a m e}_{\mathbf{S}}\right)_{2} \cdot\left(\mathbf{a m p}_{\mathrm{S}}\right)\right]^{+}\right)$, together with their calculated structures have been already published. Their fragmentation leads essentially to the $\left[\mathrm{Na} \cdot\left(\mathbf{a m e}_{\mathbf{S}}\right) \cdot\left(\mathbf{a m p}_{\mathrm{R}, \mathrm{S}}\right)\right]^{+}$and $\left[\mathrm{Na} \cdot\left(\text { ame }_{\mathbf{S}}\right)_{2}\right]^{+}$. Analogous results have been found for other metal ion/ $\alpha$-aminophosphonic mixtures investigated [10,13].

The reproducibility of the present method has been investigated changing the chirality of the reference acid ref. The results obtained using the other ref enantiomer are within ca. 10\% error and the uncertainty associated with consecutive measurements of the same system is within $5 \%$.

The average chiral resolution factors $R_{\text {chiral for }}$ the diastereomeric clusters investigated are already reported. An $\mathrm{R}_{\text {chiral }}<1$ value indicates that the heterochiral complex is more stable than the homochiral one. For $\mathrm{R}_{\text {chiral }}=1$ there is no stability differences and chiral discrimination is unattainable by this method [14].

The CID results provide only a stability order for the diastereomeric clusters and indicate that this depends upon the nature of the metal center. Molecular Mechanics MM2 Force Field calculations point to the most probable configuration for $\left[\mathrm{Na} \cdot\left(\mathbf{a m e}_{\mathbf{s}}\right)_{2} \cdot(\mathbf{a m p})\right]^{+}$as that with the reference ames molecules directly bounded to the metal center and with the amp analite placed outside [10].

\section{CONCLUDING REMARK}

Gas phase chiral molecules have been enantiodifferentiated after complexation with a chiral selector by using the REMPI-TOF and ESI-MS ${ }^{\mathrm{n}}$-CID techniques. The wavelength and mass selectivity displayed by gaseous clusters containing chiral and achiral molecules reflect the balance between stereospecific attractive (hydrogenbond, dispersive, charge exchange, etc.) and repulsive (steric) interactions operating in these systems. The spectral shifts observed in the R2PI spectra of clusters testify the role of the $\mathrm{OH} \cdots \pi$ interactions in determining their structure. The nature of the forces acting in these complexes has been explored with the support of theoretical calculations.

\section{ACKNOWLEDGEMENTS}

This work was supported by MIUR and CNR.

\section{REFERENCES}

[1] A. Latini, D. Toja, S. Piccirillo, A. Giardini Guidoni, and M. Speranza, Angew. Chem. Int. Ed. Engl. 38 (1999), 815.

[2] R. Al-Rabaa, E. Bréhéret, F. Lahmani, and A. Zehnacker, Chem. Phys. Lett. 237 (1995), 480.

[3] S. Piccirillo, C. Bosman, D. Toja, A. Giardini Guidoni, M. Pierini, A. Troiani, and M. Speranza, Angew. Chem. Int. Ed. Engl. 36 (1997), 1729.

[4] W. A. Tao, D. Zhang, E. N. Nikolaev, and R. G. Cooks, J. Am. Chem. Soc. 122 (2000), 10598; W. Y. Shen, P. S. H. Wong, and R. G. Cooks, Rapid Commun. Mass Spectrom. 11 (1997), 71.

[5] M. Sawada, Y. Takay, H. Yamada, S. Hirayama, T. Kaneda, T. Tanaka, K. Kamada, T. Mizooku, S. Takeuchi, K. Ueno, K. Hirose, Y. Tobe, and K. Naemura, J. Am. Chem. Soc. 117 (1995), 7726 and references therein.

[6] J. Laskin and J. H. Futrell, J. Phys. Chem. A 104 (2000), 8829.

[7] M. Mons, F. Piuzzi, I. Dimicoli, A. Zehnacker, and F. Lahmani, Phys. Chem. Chem. Phys. 2 (2000), 5065.

[8] A. Latini, D. Toja, A. Giardini Guidoni, A. Palleschi, S. Piccirillo, and M. Speranza, Chirality 11 (1999), 376.

[9] D. Consalvo, A. Van der Avoird, S. Piccirillo, M. Coreno, A. Giardini Guidoni, A. Mele, and M. Snels, J. Chem. Phys. 99 (1993), 8398.

[10] A. Paladini, C. Calcagni, M. Satta, T. Di Palma, M. Speranza, A. Laganà, G. Fago, A. Filippi, and A. Giardini-Guidoni, Chirality 13 (2001), 707.

[11] N. L. Allinger, Z. Q. S. Zhew, and K. Chen, J. Am. Chem. Soc. 114 (1992), 6120 
[12] D. Scuderi, A. Paladini, M. Satta, D. Catone, S. Piccirillo, M. Speranza, and A. Giardini Guidoni, Phys. Chem. Chem. Phys. 4 (2002), 4999.

[13] G. Fago, A. Filippi, A. Giardini, A. Laganà, A. Paladini, and M. Speranza, Angew. Chem. Int. Ed. 40 (2001), 4051.

[14] In previous publications (refs. [12] and [29]), according to Cooks kinetic method (ref. [6]), the freeenergy difference $\Delta(\Delta \mathrm{G})=\Delta \mathrm{G}_{\text {homo }}-\Delta \mathrm{G}_{\text {hetero of }}$ diastereomeric $[\mathbf{M} \cdot \boldsymbol{r e f} \cdot \mathbf{S}]^{+}$was derived from the $\ln \mathrm{R}_{\text {chiral }}=\Delta(\Delta \mathrm{G}) / \mathrm{RT}_{\text {eff }}$ equation, using a $\mathrm{T}_{\text {eff }}$ value estimated on the grounds of the fragmentation pattern of their $\left[\mathbf{M} \cdot(\boldsymbol{r} \boldsymbol{f})_{2} \cdot \mathbf{S}\right]^{+}$precursors. However, a rigorous theoretical analysis of Cooks method (ref. [8]) pointed out that the description of the reacting population requires the use of two different temperatures, not determinable at present. 


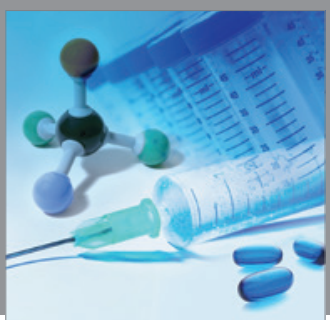

International Journal of

Medicinal Chemistry

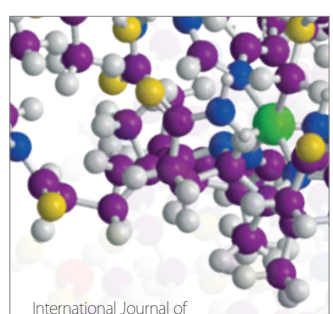

Carbohydrate Chemistry

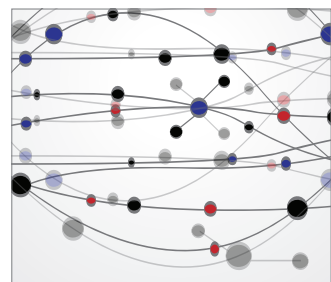

The Scientific World Journal
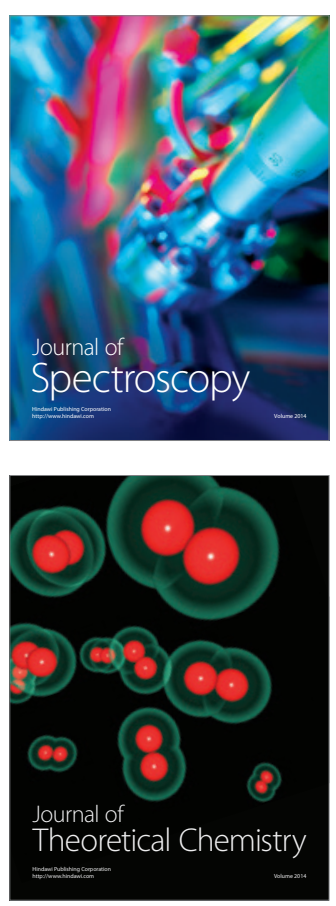
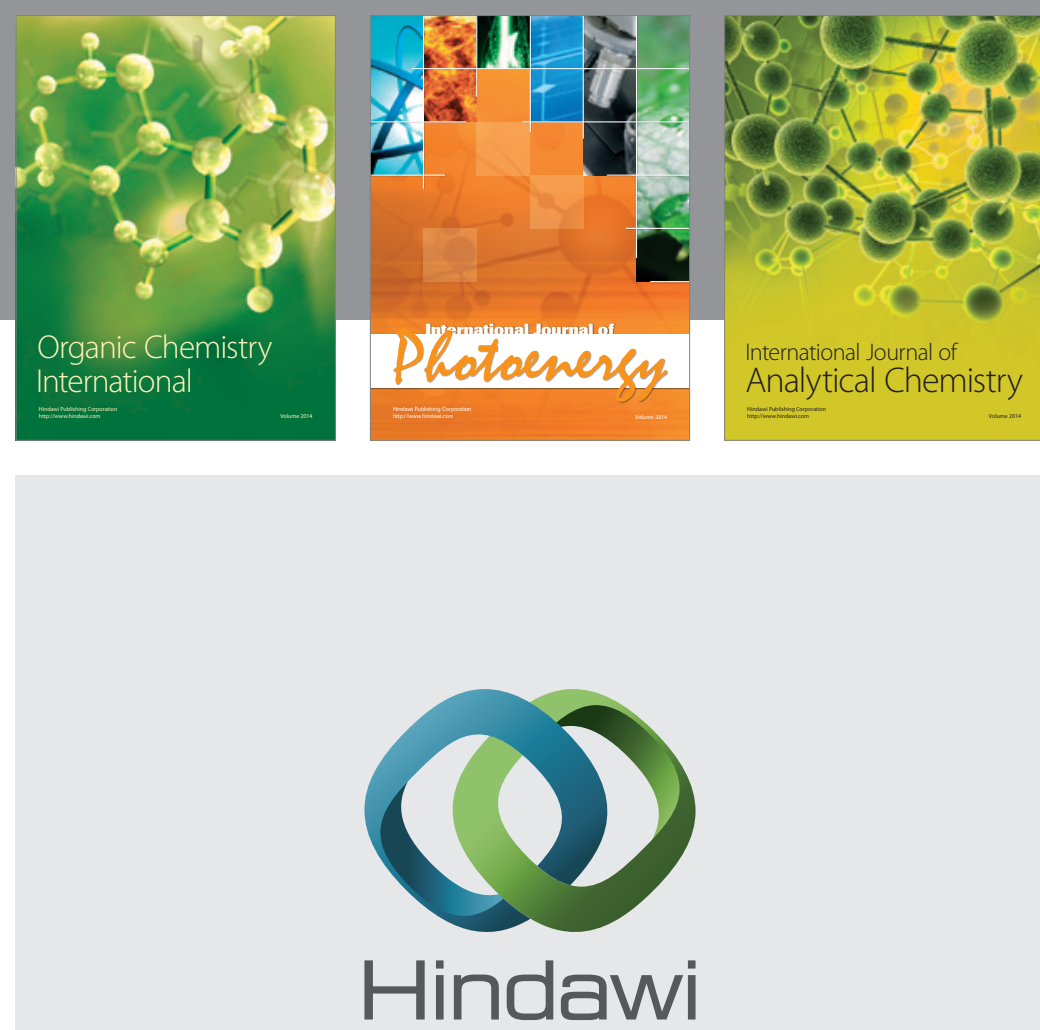

Submit your manuscripts at

http://www.hindawi.com
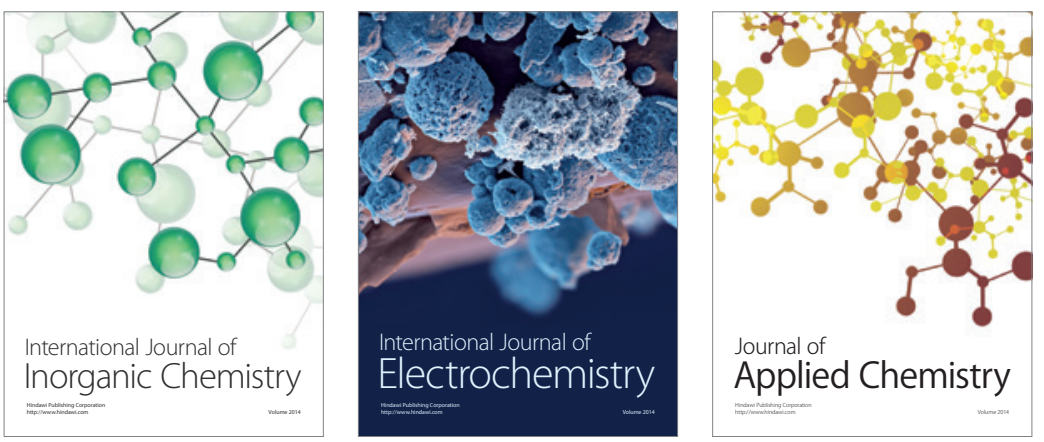

Journal of

Applied Chemistry
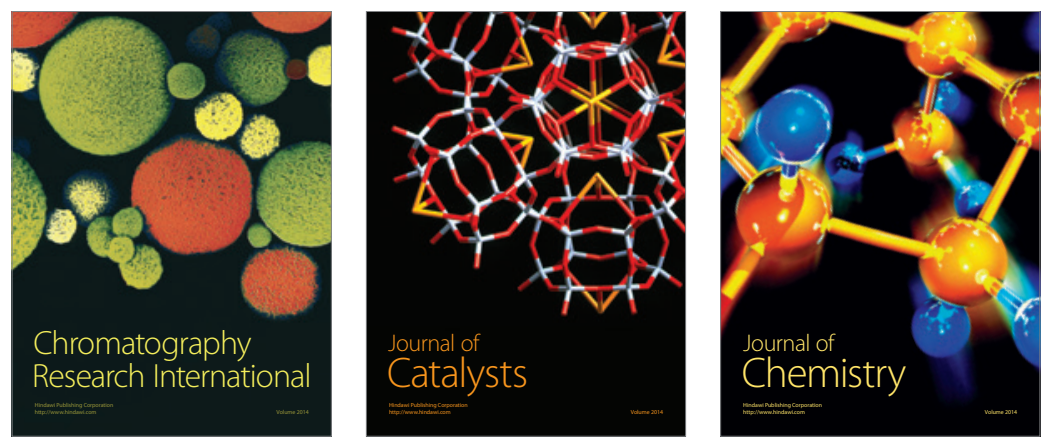
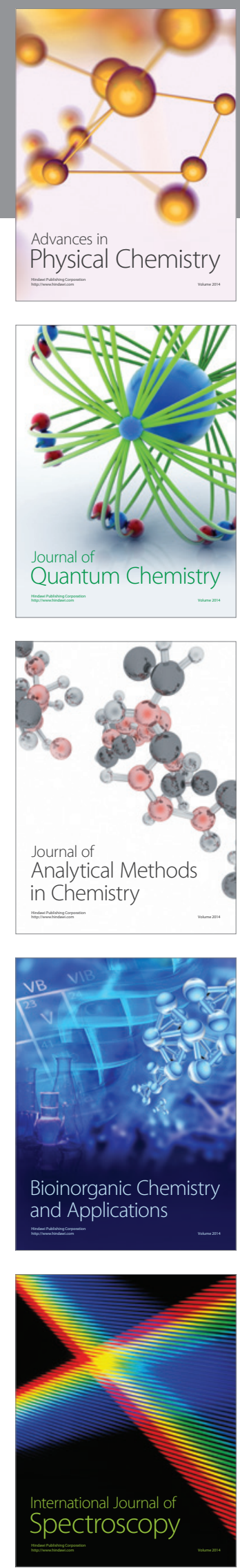